\title{
LAS INVERSIONES TÉRMICAS Y LA CONTAMINACIÓN ATMOSFÉRICA EN LA ZONA METROPOLITANA DE GUADALAJARA (MÉXICO)
}

\author{
García $^{1}$ M., Ramírez ${ }^{1}$ H., Ulloa ${ }^{1}$ H., Arias ${ }^{1}$ S., Pérez ${ }^{2}$ A. \\ ${ }^{1}$ Instituto de Astronomía y Meteorología. Universidad de Guadalajara (México) \\ ${ }^{2}$ Universidad de Santiago de Compostela (España)
}

\section{RESUMEN}

El objetivo de este trabajo es analizar el comportamiento de las inversiones térmicas y contaminantes atmosféricos en la Zona Metropolitana de Guadalajara (ZMG) durante el periodo 2005-2007. Se analizó la información de las inversiones térmicas (estación CNA colomos) y concentraciones de los contaminantes: monóxido de carbono, bióxido de nitrógeno, ozono, bióxido de azufre y partículas menores a 10 micras de las ocho estaciones de la Red de Monitoreo Atmosférico del Gobierno de Jalisco. Los resultados mostraron relación significativa entre los días con inversión térmica de gran espesor y duración con la acumulación de contaminantes, lo cual provoca que se incrementen los índices de contaminación en la zona. Los meses con presencia importante de inversiones térmicas y contaminación elevada ocurrieron durante las estaciones de invierno y primavera (noviembre-mayo). Una conclusión relevante es que las inversiones térmicas fuertes han sido factor en elevados índices de contaminación en la ZMG.

Palabras clave: Inversiones térmicas, contaminantes atmosféricos, Zona Metropolitana de Guadalajara

\section{ABSTRACT}

The objective is to analyze the behavior of thermal inversions and air pollutants in the Guadalajara Metropolitan Area during 2005-2007. Thermal inversion data (CNA colomos station) and air pollutants (carbon monoxide, nitrogen dioxide, ozone, sulfur dioxide and particulate less than 10 microns) of eight stations of 
the Air Quality Monitoring Network of the Government Jalisco were analyzed. The results indicated significant relationship between days with thermal inversion of great thickness and long time with the accumulation of pollutants, which causes an increase in the levels of pollution in the area. The months with high presence or thermal inversions and high contamination index are in winter and spring seasons (November-May). A relevant conclusion is that strong thermal inversions have influence in higher rates of air pollution in the Guadalajara Metropolitan Area.

Key words: Thermal inversions, air pollutants, Guadalajara Metropolitan Area.

\section{INTRODUCCIÓN}

Desde sus orígenes el ser humano ha emitido contaminantes a la atmósfera; sin embargo, éstos se incrementaron a gran escala durante la revolución industrial iniciada en la isla de Gran Bretaña a finales del siglo XVII, debido al remplazo del trabajo manual por maquinaria y la quema de combustibles fósiles como el carbón mineral y el petróleo con elevado contenido de azufre. Este desarrollo se expandió a Europa y América del Norte a finales del siglo XIX y durante el siglo $\mathrm{XX}$ al resto del planeta, propiciando el auge de ciudades sobrepobladas, contaminadas y, en consecuencia, efectos nocivos en la salud de la población (Ramírez et al., 2006; Segala 1999; Schwartz y Marcus, 1990).

Casos dramáticos fueron la niebla tóxica londinense (1952), el deterioro de bosques europeos por la lluvia ácida (1950-1970) y la grave situación de calidad del aire en ciudades como Tokio, Sao Paulo y México DF a finales del siglo anterior (Ramírez et al. 2008; Molina y Molina, 2005).

Los trabajos sistemáticos sobre deterioro ambiental en la República Mexicana surgen en 1980, particularmente en México DF y Toluca Estado de México. La Ciudad de México involucra una elevada densidad poblacional, vehicular e industrial producto de un acelerado desarrollo tecnológico; por tanto, no es novedad que experimente problemas ambientales, destacando la contaminación del aire. Un factor meteorológico que contribuye a ésta, son las inversiones térmicas (ITs) debido a que limitan la mezcla vertical del aire y atrapan los contaminantes debajo de la capa de inversión (Molina y Molina, 2005). En relación a ello, la cantidad de contaminantes en el aire, el espacio en el que se dispersan y los mecanismos de eliminación, son factores determinantes en el grado de contaminación (Nebel y Wright, 1999).

Desde 1980, la Zona Metropolitana de Guadalajara (ZMG en lo sucesivo) ha experimentado un crecimiento urbano, vehicular e industrial sin la planeación y control característica de las grandes ciudades, situación reflejada en los elevados 
índices de contaminación en los últimos años. Aspectos meteorológicos que contribuyen a ello son los sistemas anticiclónicos del Golfo de México y el Océano Pacífico, al causar estabilidad atmosférica e impedir la mezcla vertical del aire. Además, su relieve constituye una barrera natural para la circulación del aire impidiendo la dispersión de contaminantes en la zona (SEMARNAP/SS/GEJ, 1997).

El objetivo de este trabajo es analizar el comportamiento de las ITs y los contaminantes atmosféricos en la ZMG durante el periodo 2005-2007.

Borja et al. (1997) exponen la trascendencia de conocer la calidad del aire que se respira en las grandes ciudades debido a que la contaminación generada en ellas se vincula con una serie de enfermedades respiratorias. Los estudios pueden ser un soporte en la toma de decisiones y alertar a la población sobre posibles contingencias ambientales con suficiente antelación. Además, es relevante para considerar medidas que mitiguen y controlen los daños potenciales a la salud (Dockery et al. 1989, Schwartz et al. 1993).

\section{INVERSION TÉRMICA}

La inversión térmica (IT) es un fenómeno natural que se caracteriza por un cambio en la tendencia normal del aire al enfriarse con la altitud; cuando existe ésta, la temperatura aumenta con la altitud en determinado estrato atmosférico. Este aumento térmico puede producirse desde la superficie o a partir de una cierta altura. Se pueden presentar cuatro tipos de inversiones térmicas (de tierra, por subsidencia, por turbulencia y frontal), de tal manera que, bajo ciertas condiciones meteorológicas puede predominar alguna de ellas y contribuir a intensificar la acumulación y concentración de contaminantes en el aire (PROAIRE, 2010; Nebel y Wright, 1999; Martín 1991).

Desde la perspectiva de la salud, las ITs en sí mismas no representan riesgo para la salud humana; éstas, se caracterizan por ser más frecuentes e intensas en la temporada invernal, aunque también aparecen en el verano con menor intensidad y frecuencia. En presencia de contaminantes atmosféricos la IT aparece como una «capa de smog» de color ocre debido a la presencia de óxidos de nitrógeno y partículas suspendidas (Enger y Smith 2006; Generalitat Valenciana, 2010).

La IT se vuelve peligrosa en una zona urbana debido a que la capa de aire caliente que cubre la región impide el desarrollo de corrientes de aire ascendentes que dispersen los contaminantes. Esta situación se agrava en presencia de masas de aire de alta presión que propician una inversión prolongada durante varios días. Los contaminantes del aire en la superficie llegan a concentrarse hasta llegar a valores nocivos o letales para los seres vivos. Una IT es peligrosa porque acentúa los efectos dañinos de las islas de calor y las cúpulas de polvo que se forman sobre las áreas urbanas (Alfaro et al., 2008). 
Comúnmente, los parámetros que identifican una IT son el espesor, la intensidad, la temperatura y la hora de ruptura (figura 1 y 2). El espesor es la diferencia en metros entre la cima o tope de la IT y su base; la intensidad es la diferencia de temperaturas (en grados Celsius) de la cima y de la base; la temperatura de ruptura es el valor que se requiere alcanzar entre la base y la cima de la IT, y la hora de ruptura (h) es el instante en que se alcanza la temperatura de ruptura; ello permite estimar las condiciones idóneas de dispersión de contaminantes en el aire (Meulenert et al., 2006; Martín, 1991).

Figura 1. Parámetros que definen una inversión térmica

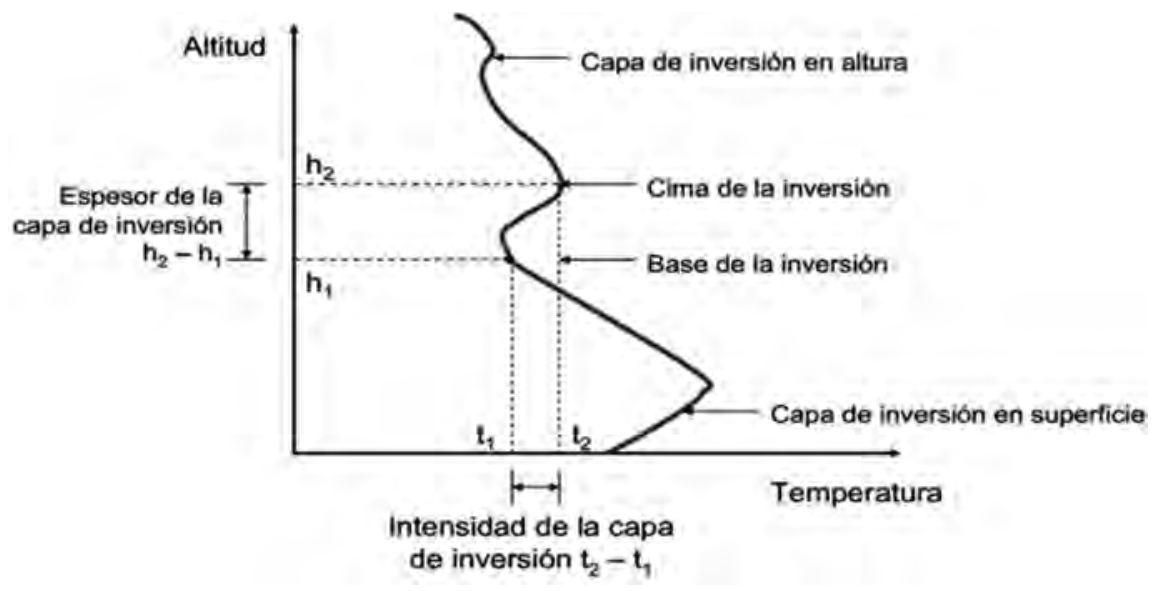

Fuente: ZMVM 2005

Figura 2. Imagen física de la inversión térmica (ZMVM 2005)

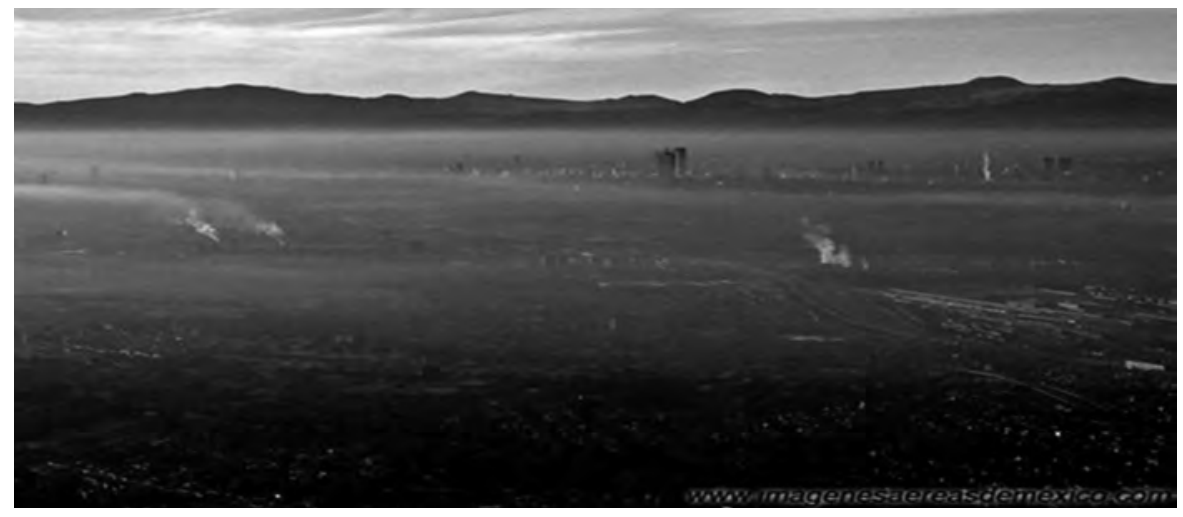

Fuente: ZMVM 2005 


\section{CASO DE ESTUDIO: LA ZONA METROPOLITANA DE GUADALAJARA (ZMG)}

\subsection{Ubicación geográfica}

Esta zona se ubica en el centro del Estado de Jalisco, en la región centro-occidente del país; sus coordenadas extremas aproximadas corresponden a latitud norte $20^{\circ} 46^{\prime} 00^{\prime \prime}$, latitud sur $20^{\circ} 32^{\prime} 08^{\prime \prime}$, longitud oriental $103^{\circ} 12^{\prime} 30^{\prime \prime}$ y longitud occidental 103⒉ $00^{\prime \prime}$, a una altitud promedio de 1,540 metros sobre nivel del mar (figura 3). Enmarca los Municipios de Guadalajara, Tlaquepaque, Tonalá y Zapopan, así como sus Cabeceras Municipales (Ramírez et al. 2008, SEMARNAP/SS/GEJ 1997).

Figura 3. Ubicación geográfica de la ZMG

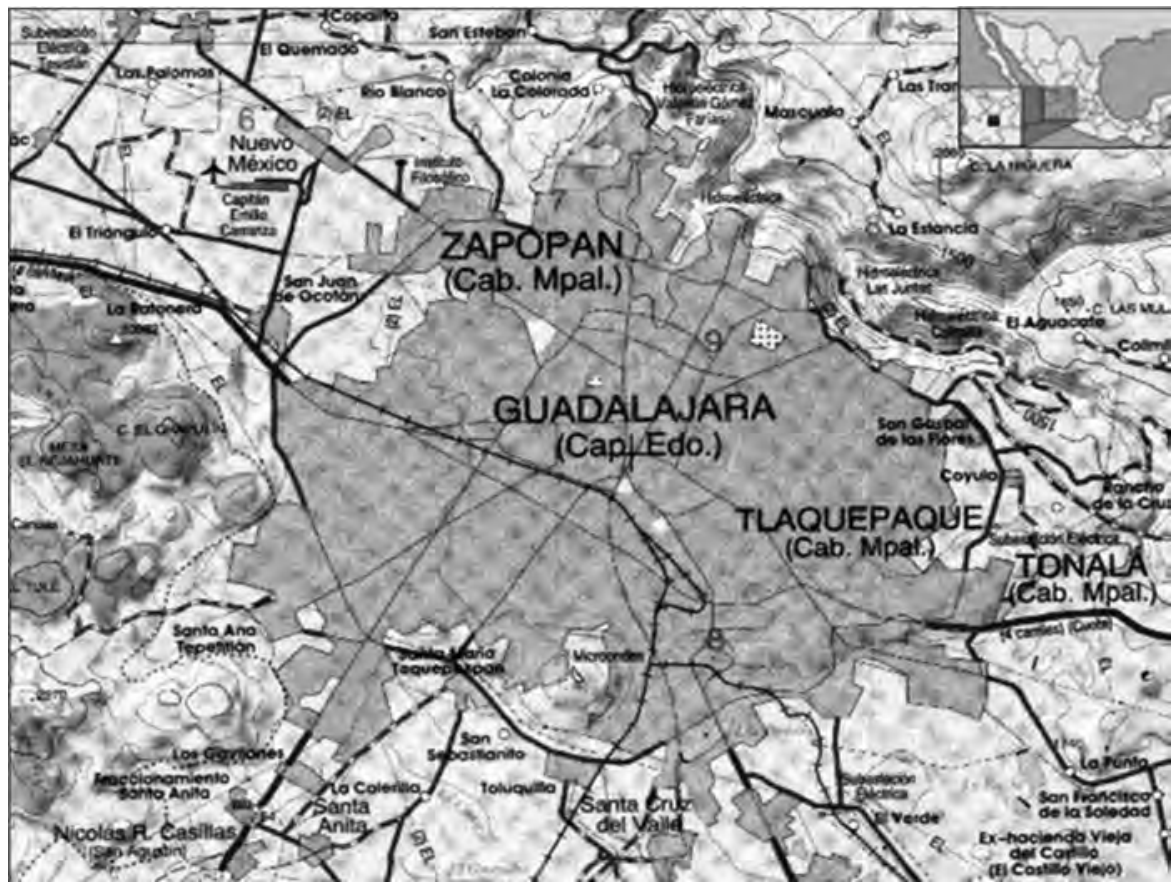

Fuente: Google imágenes

\subsection{Relieve}

La ciudad de Guadalajara se sitúa en la cuenca del valle del río Grande de Santiago, en los valles de Atemajac y la planicie de Tonalá, entre las zonas montañosas de la Sierra Madre Occidental y el eje Neovolcánico. Las montañas que circulan la zona son: al noroeste la sierra de San Esteban; al sureste, la serranía de San 
Nicolás y los conjuntos montañosos Cerro escondido-San Martín y el TapatíoLa Reina; al sur, el Cerro del cuatro-Gachupin-Santa María; y al oeste, la sierra la Primavera (figura 4). Estas sierras constituyen una barrera física natural para la circulación del viento e impiden que el aire contaminado se aleje de la zona (SEMARNAP/SS/GEJ 1997).

Figura 4. Relieve de la ZMG

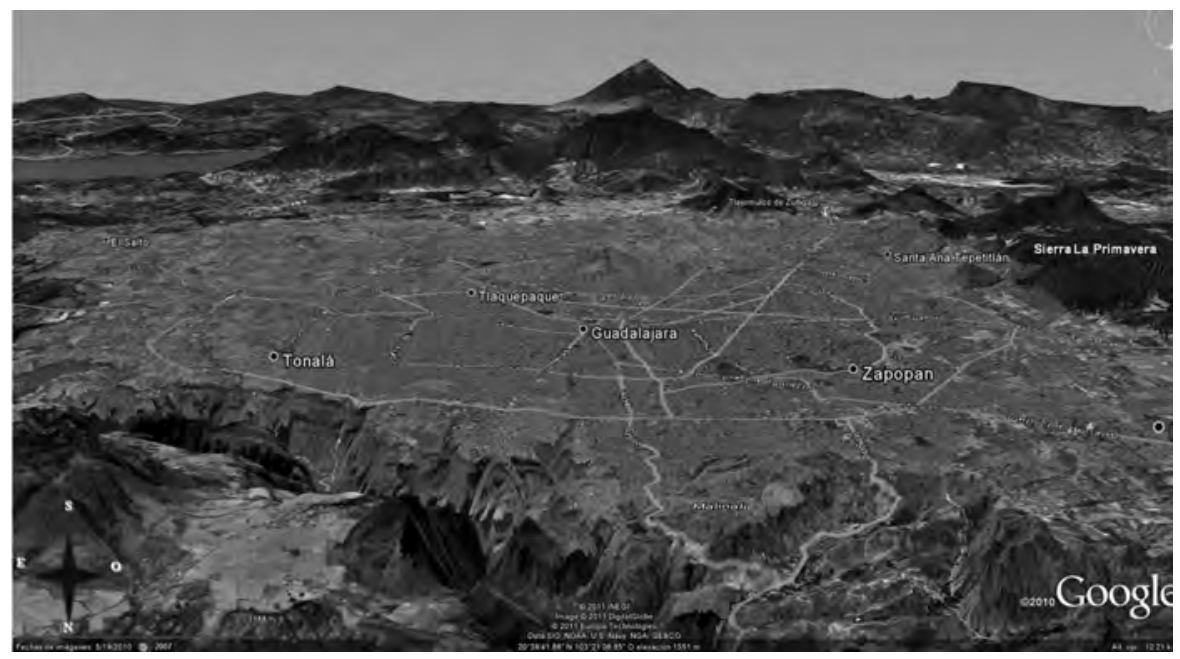

Fuente: Google imágenes

Durante los últimos años se han presentado contingencias ambientales en la ZMG producto de la mala calidad del aire, lo cual se ha relacionado con el crecimiento del parque vehicular y el desarrollo de complejos industriales tanto en el interior de la metrópoli como en las periferias. Aunado a ello, el deterioro progresivo de las áreas verdes por cambios de uso del suelo y la gran cantidad de incendios forestales en los bosques urbanos (Bosque La Primavera, entre otros), han sido factores influyentes.

Esta zona se caracteriza por presentar ITs durante la mayor parte del año; las partículas de aire se mueven con lentitud y quedan atrapadas entre la superficie y altura de cientos de metros o menos. Por tanto, un aire contaminado y con índices de calidad fuera de norma, puede propiciar la implementación de contingencias ambientales significativas.

Por lo general, las ITs se rompen antes del medio día (12:00 h) y, cuando esto sucede, la capa de smog se dispersa con el aumento del espacio en la vertical. La IT no se asocia a elevadas concentraciones del contaminante ozono dado que su mayor 
producción ocurre en horas de mayor insolación (de 14:00 a 17:00h). Las situaciones meteorológicas que provocan las ITs en la zona, aparecen con más frecuencia en los meses secos del año (de octubre a marzo) aunque también lo hacen en los meses lluviosos. En este caso, las precipitaciones ayudan a «limpiar» la atmósfera y no es tan frecuente que se eleven los índices de calidad del aire (Meulenert et al. 2006).

\section{METODOLOGÍA}

En la ZMG se cuenta con un equipo de sondeos atmosféricos que permite conocer el comportamiento de algunas magnitudes meteorológicas, tales como, la temperatura, presión atmosférica, velocidad del viento y humedad relativa. El equipo está instalado en la estación meteorológica del parque Colomos y es operado por la Comisión Nacional del Agua - Servicio Meteorológico Nacional. La información obtenida se procesa y se obtiene un perfil diario de las condiciones de la atmósfera en el lugar donde es lanzado, pudiendo determinar la existencia de una inversión térmica, su intensidad, espesor y la hora en que concluye.

Cuando no es posible obtener estos sondeos, se recurre a la utilización de técnicas de mediciones indirectas mediante los satélites meteorológicos. Éstos, desde hace muchos años realizan sondeos atmosféricos a través de microondas que miden los mismos parámetros que los equipos en tierra y los cuales han sido sometidos a rigurosas comparaciones.

En el presente trabajo se llevó a cabo un procedimiento similar al descrito, obteniéndose márgenes aceptables y adecuados para su uso. En la figura 5 se muestra un sondeo real (03/02/2006 a las 12:00 UTC [Universal Time Coordinated - 6:00 hora local]) realizado desde la estación Colomos (a) y por satélite (b) a la misma hora. Las similitudes son evidentes entre ambos perfiles y se observa la fuerte IT en ambos casos.

Se utilizaron los sondeos termodinámicos de la estación meteorológica Colomos realizados a las 12:00 UTC y la página www.ready.noaa.gov/ready/amet. html para obtener los sondeos de los días faltantes. El periodo comprendió del 01 de enero de 2005 al 31 de diciembre de 2007.

Asimismo, para conocer los índices de la calidad del aire en la ZMG, la Secretaría del Medio Ambiente y Desarrollo Sustentable del Gobierno del Estado de Jalisco (SEMADES/GEJ) opera la Red Automática de Monitoreo Atmosférico (RAMA) con ocho estaciones (área de influencia de $2 \mathrm{~km}$ entre ellas) que miden los contaminantes atmosféricos denominados criterio; éstos son: monóxido de carbono $(\mathrm{CO})$, bióxido de nitrógeno $\left(\mathrm{NO}_{2}\right)$, ozono $\left(\mathrm{O}_{3}\right)$, bióxido de azufre $\left(\mathrm{SO}_{2}\right)$ y partículas menores a 10 micras $\left(\mathrm{PM}_{10}\right)$. Con los datos de registros promedio horarios se obtuvieron las principales tendencias estadísticas correspondientes a cada año y contaminante. 
Figura 5a. Sondeo (03/02/2006-12:00 UTC)-estación Colomos

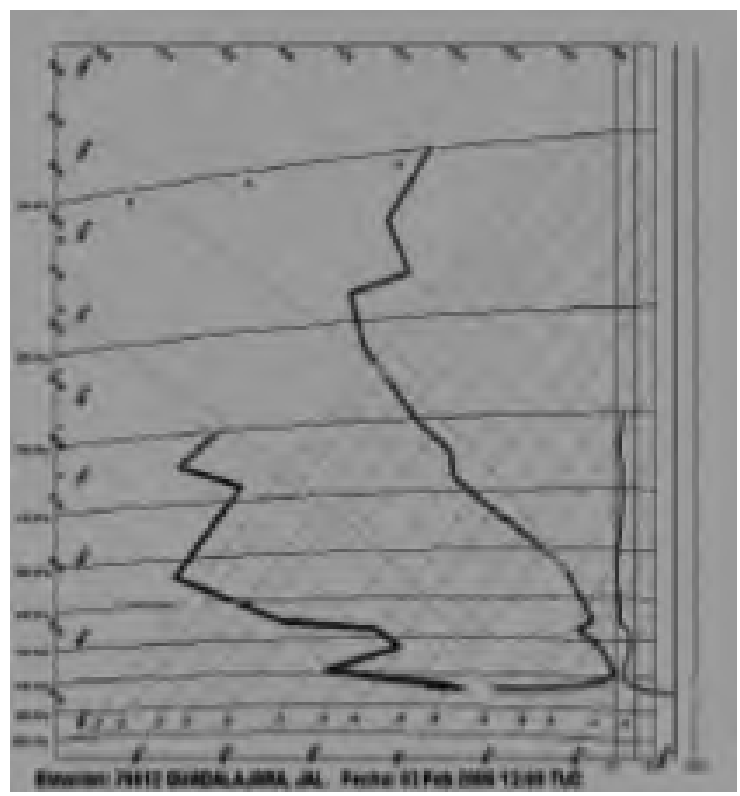

Figura 5b. Sondeo por satélite (03/02/2006-12:00 UTC)

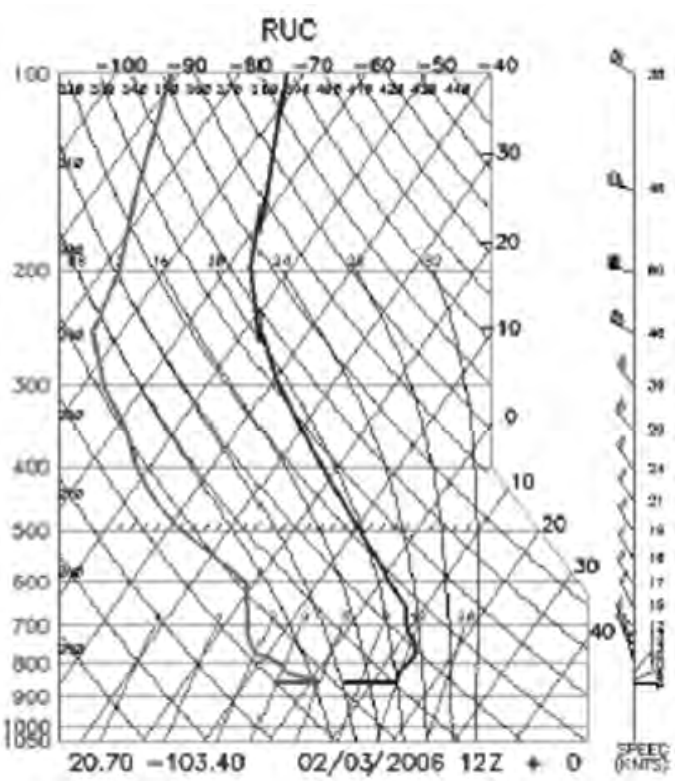

Investigaciones geográficas, nº 58, pp. 09 - 29 
Se evaluaron 390 sondeos reales y 1095 sondeos virtuales. Se consideraron tres factores relacionados con las condiciones meteorológicas que afectan a la zona: aumento de la temperatura por cada $100 \mathrm{~m}$ de altura, espesor de la capa de inversión de la superficie al techo y la hora de ruptura. Asimismo, la IT se clasificó en débil, moderada y fuerte.

IT Débil. El aumento de la temperatura con la altura es pequeño; es decir, menor de 2 a $4{ }^{\circ} \mathrm{C}$ por cada $100 \mathrm{~m}$. La atmósfera es inestable y con gran contenido de humedad. La hora de ruptura ocurre antes de las 8:00 AM.

IT Moderada. El aumento de la temperatura con la altura es elevado (mayor de $4{ }^{\circ} \mathrm{C}$ ). Su rompimiento ocurre antes del mediodía debido a condiciones meteorológicas que producen viento fuerte, aún cuando la atmósfera sea estable y seca.

IT Fuerte. Aumento significativo de la temperatura con la altura (mayor de $4{ }^{\circ} \mathrm{C}$ ). La atmósfera es predominantemente estable y seca; hay poco viento o calma y la hora de ruptura ocurre después del mediodía. Un caso especial es la «IT muy fuerte»; ocurre cuándo se exhiben características de IT fuerte pero con espesor de capa muy laminar (menor de $10 \mathrm{~m}$ ).

\section{RESULTADOS}

Se obtuvieron los días y porcentajes con inversiones térmicas por mes y año. Asimismo, se determinaron los promedios mensuales y anuales de intensidad $\left({ }^{\circ} \mathrm{C}\right)$, espesor (m) y hora de ruptura de las mismas (hora local del centro).

En el año 2005 se contabilizaron 293 días con IT (80.3\%), el 2006 mostró 218 días (59.7\%) y el 2007 evidenció 201 días (55.1\%) (figura 6a). En cuanto a la intensidad promedio anual en los tres años, resultaron gradientes de 2.3, 2.4 y 2.6 respectivamente (figura $6 \mathrm{~b}$ ). Por su parte, el espesor promedio anual fue incrementándose; $75.3 \mathrm{~m}$ en el 2005, $117.8 \mathrm{~m}$ en el 2006 y $172.5 \mathrm{~m}$ en el año 2007 (figura 6c). Las horas de ruptura promedio anual en los años 2005, 2006 y 2007 ocurrieron a las 16:00, 15:00 y 15:00 horas respectivamente (figura 6d).

Durante los tres años, el número de ITs fue más importante en los meses de invierno y primavera (noviembre-mayo), justo antes del temporal de lluvias (figura 7a). Las intensidades promedio mensuales mostraron un comportamiento similar a las frecuencias. De noviembre a mayo se presentaron las mayores intensidades en el gradiente de temperatura (figura $7 \mathrm{~b}$ ). En cuanto al espesor, se reportaron valores muy heterogéneos, aunque se observó un aumento del 2005 al 2007 (figura 7c). En este orden, en el intervalo de noviembre a mayo se presentaron las horas de ruptura más prolongadas (figura 7d). 
Figura 6. a) Frecuencia, b) Intensidad, c) Espesor, d) Hora de ruptura

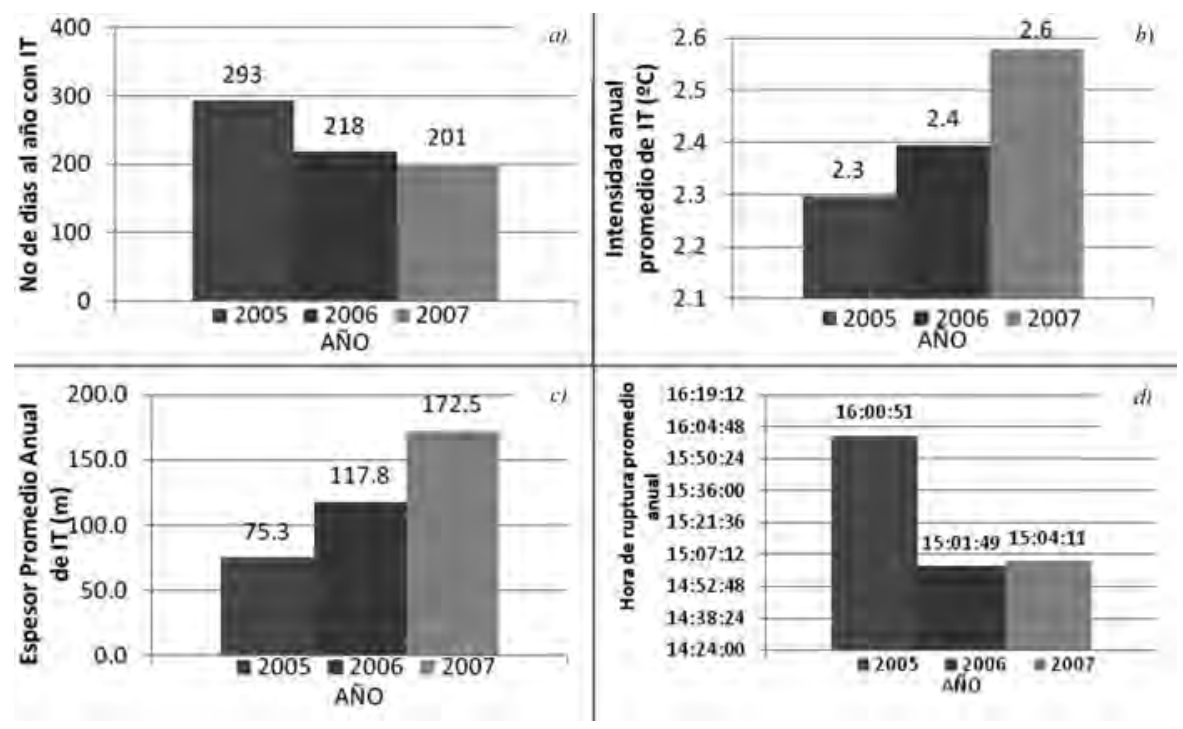

Por lo que respecta a las intensidades, en los tres años el rango de variación máximo ocurrió en los meses de noviembre a mayo, mientras que, en los meses del periodo lluvioso el rango se reduce. Con respecto a los espesores, prácticamente durante todos los meses del año 2005 y 2006 presentaron rangos entre 20 y 100 metros; sin embargo, junio, julio y diciembre del 2006 aumentaron de manera considerable entre 500 y 1000 m, manteniéndose el 2007 en rangos de hasta $250 \mathrm{~m}$ (tabla 1).

Por otra parte, como se indica en la tabla 2, en el año 2005 se presentaron 293 ITs de las cuales 45 con intensidad entre 0.1 y $1.0^{\circ} \mathrm{C}$, 80 en el rango de 1.1 a $2.0^{\circ} \mathrm{C}, 100$ eventos entre 2.1 y $3^{\circ} \mathrm{C}$. En relación al rango de 3.1 a $4^{\circ} \mathrm{C}$, la frecuencia fue de 18 veces; mientras que, con intensidad fuerte (mayor a $4^{\circ} \mathrm{C}$ ) la ocurrencia fue de 50.

En el caso del año 2006, se reportaron 218 inversiones térmicas, de las cuales 38 fueron con intensidad entre 0.1 y $1.0^{\circ} \mathrm{C}, 50$ en el rango de 1.1 y $2.0^{\circ} \mathrm{C}$, 52 eventos en el intervalo de $2.1-3.0^{\circ} \mathrm{C}$ y 20 entre 3.1 y $4^{\circ} \mathrm{C}$; por otra parte, la intensidad superior a los $4.0^{\circ} \mathrm{C}$ se presentó en 58 sucesos.

Similarmente, el año 2007 reportó 201 ITs; de ellas, 55 eventos con intensidad entre 0.1 y $1.0^{\circ} \mathrm{C}, 41$ con intensidad de 1.1 a $2.0^{\circ} \mathrm{C}, 27$ sucesos en el rango de 2.1 a $3.0{ }^{\circ} \mathrm{C}$ y 19 con 3.1 y $4{ }^{\circ} \mathrm{C}$. Asimismo, 59 ocurrieron con intensidad superior a $4.0^{\circ} \mathrm{C}$. 
Figura 7. a) Frecuencia mensual, b) Intensidad, c) Espesor y d) Hora de ruptura de ITs
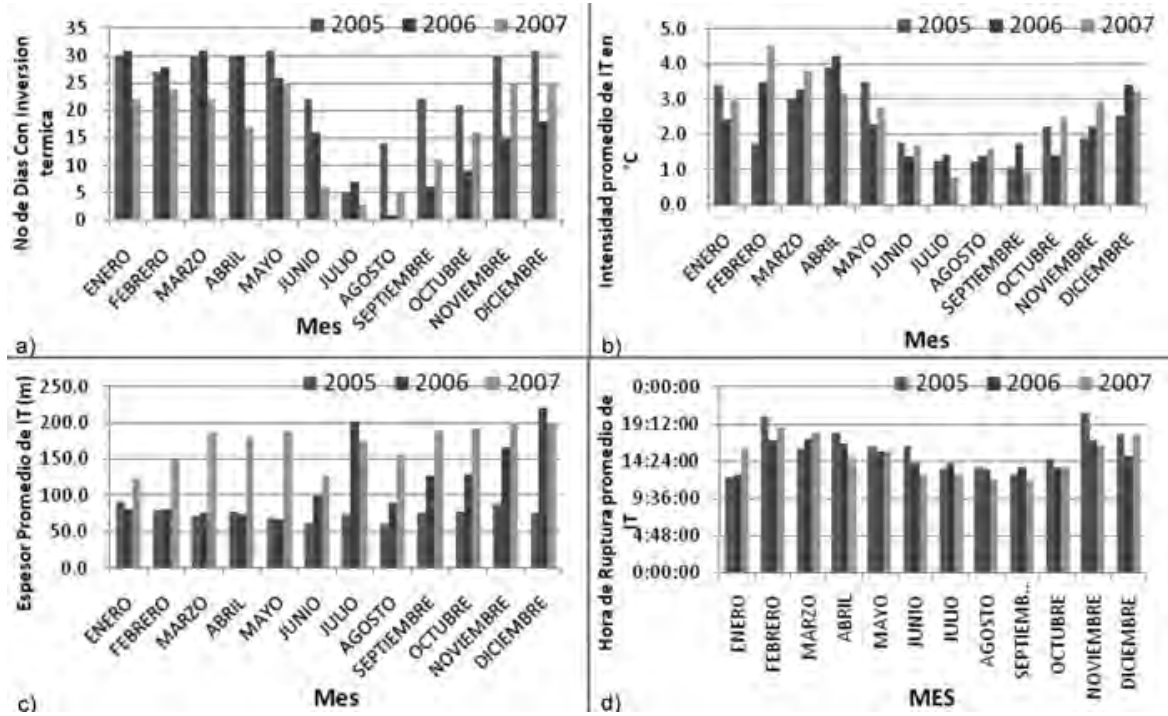

c)

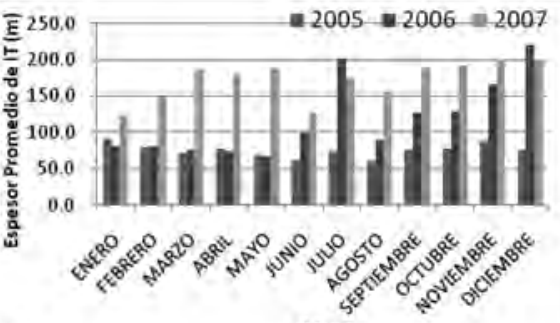

Mes

d)

MES

En cuanto al análisis anual de las ITs, en el año 2005 las intensidades de bajas a moderadas fueron las más importantes, mientras que, en los años 2006 y 2007 las más significativas fueron las moderadas y fuertes (figura 8). En el estudio mensual los valores más trascendentes ocurren entre noviembre y mayo (análogo a otros parámetros de la inversión), descendiendo en los meses lluviosos.

Tabla 1. Intensidad $\left({ }^{\circ} \mathrm{C}\right)$ y espesor $(\mathrm{m})$ de las ITs por mes y año (máximos-mínimos)

\begin{tabular}{|c|c|c|c|c|c|}
\hline & Mes & Intensidad máxima & Intensidad mínima & Espesor máxima & Espesor mínimo \\
\hline \multirow{13}{*}{ 苂 } & Enero & 7 & 0 & 127 & 40 \\
\hline & Febrero & 4 & 1 & 106 & 38 \\
\hline & Marzo & 6 & 1 & 111 & 36 \\
\hline & Abril & 10 & 2 & 106 & 30 \\
\hline & Mayo & 9 & 1 & 98 & 32 \\
\hline & Junio & 3 & 0 & 277 & 24 \\
\hline & Julio & 4 & 0 & 99 & 48 \\
\hline & Agosto & 2 & 0 & 109 & 41 \\
\hline & Septiembre & 2 & 0 & 99 & 30 \\
\hline & Octubre & 5 & 0 & 122 & 42 \\
\hline & Noviembre & 3 & 1 & 137 & 44 \\
\hline & Diciembre & 6 & 1 & 123 & 18 \\
\hline & Anual & 10 & 0 & 277 & 18 \\
\hline
\end{tabular}

Investigaciones geográficas, $n^{\circ} 58$, pp. 09 - 29 


\begin{tabular}{|c|c|c|c|c|c|}
\hline \multirow{13}{*}{ ¿̊丶 } & Enero & 6.5 & 0.2 & 121 & 42 \\
\hline & Febrero & 7.2 & 1 & 111 & 34 \\
\hline & Marzo & 7.8 & 1.2 & 126 & 30 \\
\hline & Abril & 7.7 & 1.3 & 111 & 27 \\
\hline & Mayo & 6.3 & 0.3 & 109 & 39 \\
\hline & Junio & 5.6 & 0.3 & 412 & 44 \\
\hline & Julio & 2.4 & 0.6 & 425 & 50 \\
\hline & Agosto & 1.4 & 1.4 & 89 & 89 \\
\hline & Septiembre & 4.1 & 0.2 & 197 & 59 \\
\hline & Octubre & 2.7 & 0.6 & 263 & 30 \\
\hline & Noviembre & 4.6 & 0.1 & 261 & 28 \\
\hline & Diciembre & 6 & 0.3 & 1033 & 29 \\
\hline & Anual & 7.8 & 0.1 & 1033 & 27 \\
\hline \multirow{13}{*}{ ¿্ণ } & Enero & 6.2 & 0.3 & 259 & 29 \\
\hline & Febrero & 9.7 & 0.6 & 264 & 29 \\
\hline & Marzo & 11 & 0.3 & 259 & 29 \\
\hline & Abril & 9 & 0.3 & 223 & 49 \\
\hline & Mayo & 8 & 0.3 & 217 & 49 \\
\hline & Junio & 4.2 & 0.2 & 262 & 40 \\
\hline & Julio & 1.6 & 0.2 & 217 & 98 \\
\hline & Agosto & 2.3 & 0.9 & 211 & 40 \\
\hline & Septiembre & 1.9 & 0.3 & 221 & 99 \\
\hline & Octubre & 5.7 & 0.3 & 241 & 79 \\
\hline & Noviembre & 7.8 & 0.4 & 238 & 48 \\
\hline & Diciembre & 8.8 & 0.3 & 243 & 59 \\
\hline & Anual & 11 & 0.2 & 264 & 29 \\
\hline
\end{tabular}


Las inversiones térmicas y la contaminación atmosférica en la zona metropolitana...

Tabla 2. Número de ITs de acuerdo a la intensidad $\left({ }^{\circ} \mathrm{C}\right)$ de ocurrencia

\begin{tabular}{|c|c|c|c|c|c|c|c|}
\hline & Mes & $0.1-1.0$ & $1.1-2.0$ & $2.1-3.0$ & $3.1-4.0$ & $>4.0$ & Mensual \\
\hline \multirow{13}{*}{ 岱 } & Enero & 5 & 5 & 3 & 2 & 15 & 30 \\
\hline & Febrero & 8 & 12 & 5 & 0 & 2 & 27 \\
\hline & Marzo & 2 & 4 & 12 & 6 & 6 & 30 \\
\hline & Abril & 0 & 2 & 14 & 4 & 10 & 30 \\
\hline & Mayo & 0 & 4 & 16 & 1 & 10 & 31 \\
\hline & Junio & 5 & 5 & 12 & 0 & 0 & 22 \\
\hline & Julio & 2 & 2 & 0 & 1 & 0 & 5 \\
\hline & Agosto & 6 & 7 & 1 & 0 & 0 & 14 \\
\hline & Septiembre & 11 & 9 & 2 & 0 & 0 & 22 \\
\hline & Octubre & 3 & 9 & 5 & 1 & 3 & 21 \\
\hline & Noviembre & 1 & 12 & 16 & 1 & 0 & 30 \\
\hline & Diciembre & 2 & 9 & 14 & 2 & 4 & 31 \\
\hline & Anual & 45 & 80 & 100 & 18 & 50 & 293 \\
\hline \multirow{13}{*}{$\begin{array}{l}\text { ¿ } \\
\text { ঠ }\end{array}$} & Enero & 5 & 8 & 13 & 1 & 4 & 31 \\
\hline & Febrero & 1 & 4 & 8 & 5 & 10 & 28 \\
\hline & Marzo & 0 & 11 & 7 & 2 & 11 & 31 \\
\hline & Abril & 0 & 4 & 5 & 5 & 16 & 30 \\
\hline & Mayo & 10 & 3 & 5 & 4 & 4 & 26 \\
\hline & Junio & 9 & 4 & 2 & 0 & 1 & 16 \\
\hline & Julio & 2 & 3 & 2 & 0 & 0 & 7 \\
\hline & Agosto & 0 & 1 & 0 & 0 & 0 & 1 \\
\hline & Septiembre & 3 & 1 & 1 & 0 & 1 & 6 \\
\hline & Octubre & 4 & 2 & 3 & 0 & 0 & 9 \\
\hline & Noviembre & 2 & 5 & 3 & 3 & 2 & 15 \\
\hline & Diciembre & 2 & 4 & 3 & 0 & 9 & 18 \\
\hline & Anual & 38 & 50 & 52 & 20 & 58 & 218 \\
\hline
\end{tabular}




\begin{tabular}{|c|c|c|c|c|c|c|c|}
\hline \multirow{13}{*}{ 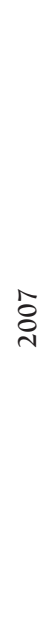 } & Enero & 3 & 5 & 4 & 4 & 6 & 22 \\
\hline & Febrero & 2 & 3 & 5 & 2 & 12 & 24 \\
\hline & Marzo & 6 & 5 & 1 & 2 & 8 & 22 \\
\hline & Abril & 6 & 1 & 2 & 2 & 6 & 17 \\
\hline & Mayo & 12 & 4 & 1 & 1 & 7 & 25 \\
\hline & Junio & 3 & 1 & 1 & 0 & 1 & 6 \\
\hline & Julio & 2 & 1 & 0 & 0 & 0 & 3 \\
\hline & Agosto & 1 & 3 & 1 & 0 & 0 & 5 \\
\hline & Septiembre & 7 & 4 & 0 & 0 & 0 & 11 \\
\hline & Octubre & 4 & 4 & 2 & 2 & 4 & 16 \\
\hline & Noviembre & 6 & 6 & 5 & 0 & 8 & 25 \\
\hline & Diciembre & 3 & 4 & 5 & 6 & 7 & 25 \\
\hline & Anual & 55 & 41 & 27 & 19 & 59 & 201 \\
\hline
\end{tabular}

Figura 8. Distribución de intensidad y frecuencia anual de ITs

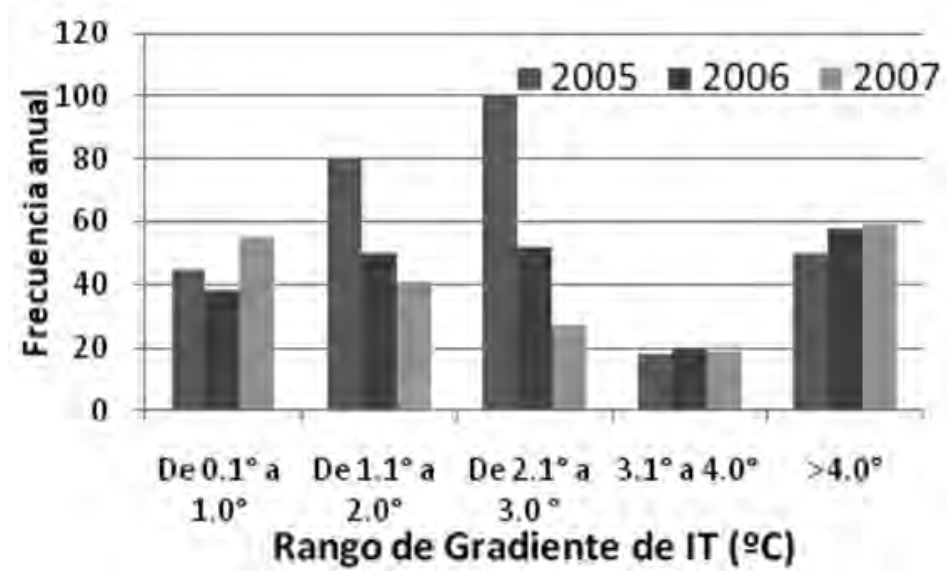

En relación a los contaminantes atmosféricos, los resultados mostraron un comportamiento variable durante el año y entre los años analizados. No obstante, se evidenció que las $\mathrm{PM}_{10}$ es el contaminante con la mayor trascendencia seguido del $\mathrm{O}_{3}, \mathrm{NO}_{2}, \mathrm{CO}$ y $\mathrm{SO}_{2}$. Las zonas más afectadas correspondieron al cuadrante sureste de la ZMG con los registros más elevados, tanto en sus concentraciones máximas, medias aritméticas y modas. Esta zona es la más recurrente durante los años analizados.

Los resultados anuales mostraron que un porcentaje importante de días superan las Normas Oficiales Mexicanas (NOM) de concentración de contaminan- 
tes. Los meses de abril a junio reportaron elevada concentración de $\mathrm{O}_{3}$ y $\mathrm{CO}$, mientras que, de diciembre a marzo reflejaron intensa concentración de $\mathrm{PM}_{10}$, $\mathrm{NO}_{2}, \mathrm{CO}$ y $\mathrm{SO}_{2}$, debido a las bajas temperaturas que prolongan la duración de las inversiones térmicas y a la poca humedad del ambiente (no permitiendo su dispersión).

El análisis por contaminante observó lo siguiente:

Monóxido de Carbono (CO)-NOM-021-SSAl-1993: 11 ppm-(DOF-23/12/1994)

Las concentraciones promedio mensuales se mantuvieron con variaciones estacionales y con tendencia constante durante el periodo de estudio. Los valores se mantuvieron por debajo de las normas EPA ( 9 ppm) y NOM, siendo su promedio mensual de $1.942 \pm 0.647 \mathrm{ppm}$ y con un rango de variación entre 0.000$4.883 \mathrm{ppm}$.

De manera similar, las modas mensuales presentaron variaciones estacionales con tendencia a mantener las concentraciones constantes y dentro de las normas EPA y NOM. Su valor promedio fue $1.129 \pm 0.502 \mathrm{ppm}$ y con un rango de 0.000 a 4.400 ppm.

Por otra parte, las concentraciones máximas mensuales mostraron valores por encima de la NOM en la mayoría del periodo; además, se observaron picos que alcanzaron valores cercanos a $55.000 \mathrm{ppm}$ (cinco veces la NOM y nueve la EPA), irregularidades que constituyeron factores de riesgo para la población. Estos eventos se registraron en el periodo más seco del año (marzo a junio), justo antes del periodo de las precipitaciones. Su valor promedio fue de $9.166 \pm 6.021 \mathrm{ppm}$. El rango de valores máximos mensuales se mantuvo entre 0.000-53.600 ppm (tabla 3). Las zonas más afectadas fueron centro y sureste de la ZMG, observando que la contaminación generada por el $\mathrm{CO}$ es considerada significativa únicamente en los momentos de picos máximos.

Bióxido de Nitrógeno ( $\mathrm{NO}_{2}$ )-NOM-023-SSA1-1993: 0.21 ppm-(DOF-23/12/1994)

La mayoría de las concentraciones promedio mensual reportaron valores dentro de la norma EPA (0.25 ppm) y NOM; sin embargo, se muestran algunas irregularidades de valores por encima de la EPA (0.05-0.10 ppm). Su valor fue de 0.034 $\pm 0.011 \mathrm{ppm}$, con un rango de variación entre 0.000-0.089 ppm.

Las modas mensuales presentaron variaciones estacionales con tendencia hacia concentraciones constantes durante el periodo y por debajo de las normas EPA y NOM. El promedio fue de $0.025 \pm 0.012 \mathrm{ppm}$, con el rango de valores entre 0.000-0.136 ppm. 
Las concentraciones máximas mensuales mostraron variación importante (0.000-0.526 ppm); éstas, se presentaron tanto en invierno como verano, no registrando un comportamiento cíclico. Su valor promedio fue de $0.114 \pm 0.071$ ppm y aunque no se observó tendencia dominante hacia un área determinada, la contaminación por $\mathrm{NO}_{2}$ es un problema grave de la ZMG (tabla 3).

Ozono (O $\left.{ }_{3}\right)$-NOM-020-SSA1-1993: 0.11 ppm-(DOF-23/12/1994)Modificación DOF-30/10/2002

Las concentraciones promedio mensuales mostraron variaciones estacionales con tendencia constante durante el periodo y con valores por debajo de la NOM. $\mathrm{Su}$ valor promedio fue $0.023 \pm 0.008 \mathrm{ppm}$, mientras que, el rango osciló de 0.000 a 0.053 ppm.

De igual manera, las modas mensuales presentaron variaciones estacionales y con valores dentro de la norma. Su valor promedio fue $0.009 \pm 0.006 \mathrm{ppm}$, mientras que el rango varió desde 0.000 a 0.047 ppm.

En la mayoría del periodo analizado las concentraciones máximas mensuales estuvieron fuera de norma y con ligera tendencia a incrementarse, presentándose variaciones significativas desde 0.000 hasta 0.650 ppm. Los picos máximos ocurrieron en la época de estiaje y en verano con mayor insolación, idóneo para las reacciones químicas de transformación $\mathrm{a}_{3}$ mediante los contaminantes primarios. Su valor promedio fue $0.110 \pm 0.044 \mathrm{ppm}$. El centro y sureste fue el área más afectada; y aunque la contaminación es considerada moderada, representa un factor de riesgo para la salud de la población, sobre todo en sus concentraciones máximas (tabla 3).

Partículas menores a 10 micras $\left(\mathrm{PM}_{10}\right)$-NOM-025-SSA1-1993: $150 \mu \mathrm{g} / \mathrm{m}^{3}-$

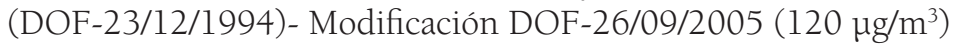

Las concentraciones medias mensuales presentaron valores entre 0 y $156 \mu \mathrm{g} / \mathrm{m}^{3}$; la

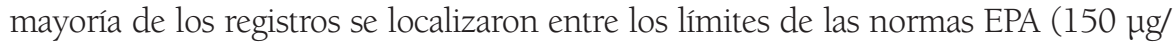
$\mathrm{m}^{3}$ ) y NOM. La zona mantiene estos niveles sin mostrar descenso; factor para inferir que es el contaminante más importante. Su valor promedio fue $50.92 \pm 20.22 \mu \mathrm{g} / \mathrm{m}^{3}$.

Las modas mensuales presentaron variaciones estacionales con tendencia a mantener las concentraciones constantes durante el periodo de estiaje (septiembre a mayo); la mayoría de los valores se encontraron por debajo de la norma EPA y NOM. Su promedio fue $35.49 \pm 33.40 \mu \mathrm{g} / \mathrm{m}^{3}$, siendo su rango de valores de 0.00 a $499.90 \mu \mathrm{g} / \mathrm{m}^{3}$.

Las concentraciones máximas se mantuvieron fuera de norma con un rango de $0-500 \mu \mathrm{g} / \mathrm{m}^{3}$, manteniendo un comportamiento constante. Su valor prome- 
dio fue $265.12 \pm 108.63 \mu \mathrm{g} / \mathrm{m}^{3}$. Los episodios extremos se localizaron al sur y sureste de la zona durante todos los años (tabla 3). Las $\mathrm{PM}_{10}$ es el principal contaminante atmosférico en la ZMG, un problema de contaminación ambiental y factor de riesgo para la salud de la población.

Bióxido de azufre $\left(\mathrm{SO}_{2}\right)$-NOM-022-SSA1-1993: 0.13 ppm- (DOF-23/12/1994)

Las concentraciones promedio mensuales variaron entre 0.000 y 0.068 ppm; su valor promedio fue de $0.009 \pm 0.005 \mathrm{ppm}$, no rebasando la norma de la EPA (0.14 ppm) y NOM (0.13 ppm).

Las modas mensuales presentaron variaciones estacionales con tendencia a mantener sus concentraciones constantes en el periodo analizado (valores por debajo de la EPA y NOM). Su valor promedio fue similar al promedio $(0.007 \pm$ $0.003 \mathrm{ppm})$, mientras que, el rango fue más elevado (0.000-0.052 ppm).

La mayoría de las concentraciones máximas mensuales presentaron valores por debajo de las normas EPA y NOM. Las máximas evidenciaron variaciones significativas de 0.000 a $0.534 \mathrm{ppm}$, siendo su valor promedio de $0.049 \pm 0.056$ ppm. El centro, sur y sureste fue el área más afectada; aun así, el $\mathrm{SO}_{2}$ no se identificó como un contaminante de riesgo para la salud de la población de la ZMG (tabla 3). En este estudio se identificó que el $\mathrm{SO}_{2}$ es el único contaminante que no rebasa los valores máximos normativos, por lo que no constituye riesgos para la salud de la población.

Tabla 3. Media, desviación estándar, máximas y mínimas de los contaminantes atmosféricos en la ZMG

\begin{tabular}{|c|c|c|c|c|c|c|c|c|c|c|c|c|}
\hline & \multicolumn{5}{|c|}{ Medias } & \multicolumn{5}{c|}{ Modas } & \multicolumn{4}{c|}{ Máximos } \\
\hline & $\mathrm{X}$ & $\mathrm{S}$ & Máximo & Mínimo & $\mathrm{X}$ & $\mathrm{S}$ & Máximo & Mínimo & $\mathrm{X}$ & $\mathrm{S}$ & Máximo & Mínimo \\
\hline $\mathrm{CO}$ & 1.942 & 0.647 & 4.883 & 0.316 & 1.129 & 0.502 & 4.400 & 0.100 & 9.166 & 6.021 & 53.600 & 1.800 \\
\hline $\mathrm{NO}_{2}$ & 0.034 & 0.011 & 0.081 & 0.006 & 0.025 & 0.012 & 0.136 & 0.002 & 0.114 & 0.071 & 0.526 & 0.019 \\
\hline $\mathrm{O}_{3}$ & 0.023 & 0.008 & 0.053 & 0.008 & 0.009 & 0.006 & 0.047 & 0.001 & 0.110 & 0.044 & 0.650 & 0.026 \\
\hline $\mathrm{PM}_{10}$ & 50.92 & 20.22 & 156.00 & 8.54 & 34.49 & 33.40 & 499.900 & 0.100 & 265.12 & 108.63 & 500.00 & 37.200 \\
\hline $\mathrm{SO}_{2}$ & 0.009 & 0.005 & 0.068 & 0.001 & 0.007 & 0.003 & 0.052 & 0.001 & 0.049 & 0.056 & 0.534 & 0.001 \\
\hline
\end{tabular}

\section{DISCUSIÓN Y CONCLUSIONES}

El análisis de las inversiones térmicas y de los contaminantes atmosféricos en el ZMG muestran que se empiezan a exhibir riesgos ambientales similares a la Ciudad de México, aún cuándo existen diferencias significativas entre sus características climáticas y topográficas. 
Durante el periodo de análisis se presentaron contingencias ambientales importantes, particularmente, asociadas a factores meteorológicos tales como, inversiones térmicas de gran espesor (cientos de metros) y duración (hora de ruptura a las 14:00 y/o 15:00 horas), estabilidad atmosférica (vientos en calma) y grandes cantidades de emisiones contaminantes concentrados en la zona al no encontrar las condiciones propicias de dispersión por los factores mencionados.

Asimismo, el parque vehicular en la ZMG ha crecido de manera significativa y gran parte cuenta con más de 10 años de uso, predominando la ausencia de mantenimiento, lo cual implica un aporte adicional de contaminantes.

De hecho, los resultados del presente trabajo demuestran que las concentraciones máximas de todos los contaminantes rebasan la norma (excepto el $\mathrm{SO}_{2}$ ). Asimismo, las medias y modas se mantienen por debajo de la NOM y EPA; a pesar de ello, los hallazgos encontrados constituyen factores potenciales de riesgo para la salud de la población (OMS 0.007 ppm).

Un estudio realizado por la CEPAL para otras áreas de América latina, reportó que la ubicación geográfica sumada a factores meteorológicos constituye una característica que explica el deterioro de la calidad del aire en las ciudades. Una orografía abrupta con la existencia de cerros o colinas es un factor limitante a la ventilación y dispersión de los contaminantes. A esto se suman factores como la velocidad de los vientos y la existencia de limitaciones a la dispersión vertical por efecto de IT que también dificultan la difusión de los contaminantes. La altura donde se produce la mezcla de contaminantes es variable según las horas del día y las estaciones del año; no obstante, la altura de la capa queda afectada por la IT. Así, la sinergia «geografía-factores meteorológicos» limita la dispersión de contaminantes y contribuyen al deterioro de la calidad del aire (Nicod y Lizuka, 2000). En este sentido, Céspedes (2005) mediante un estudio realizado en Cochabamba (Bolivia) reportó que factores meteorológicos como el transporte y dispersión, la turbulencia y las limitaciones de dispersión vertical por efecto de las capas de inversión térmica, son elementos que contribuyen a la contaminación del aire en las ciudades.

De acuerdo a lo expuesto en el presente estudio se puede concluir lo siguiente:

Las inversiones térmicas débiles ocurren todo el año y dominan en la temporada de lluvias (mitad de mayo a finales de octubre); las ITs intensas son escasas o no ocurren.

Las ITs más intensas (muy fuertes, fuertes y moderadas) se concentran en los meses de estiaje (de finales de octubre a inicios de mayo); asimismo, la frecuencia mayor de inversiones intensas ocurre en febrero. 
En cuanto a los contaminantes atmosféricos, las $\mathrm{PM}_{10}$ mostraron las concentraciones promedio mensuales más elevadas, identificándose como el contaminante principal, un problema eminente de contaminación ambiental y de riesgos para la salud.

La contaminación por CO es considerada significativa (riesgos para la salud) únicamente en los picos de máxima concentración.

En cuanto al $\mathrm{NO}_{2}$ sus concentraciones máximas son las que más frecuentemente se mantienen por encima de la norma en toda el área de estudio.

$\mathrm{El} \mathrm{O}_{3}$ es moderado en la zona, sin embargo, la exposición a altas concentraciones ocasionales representa un riesgo para la población.

Las concentraciones de $\mathrm{SO}_{2}$ se mantienen por debajo de la normatividad, por lo que no representa riesgos para la población.

Las zonas continuamente más afectadas son el centro, sur y sureste de la ZMG con valores extremos eventuales en el resto.

Por último, es inevitable destacar que el crecimiento acelerado de la ZMG ha propiciado que las estaciones de monitoreo mencionadas se hayan hecho rápidamente insuficientes para cubrir adecuadamente el área. Otra situación preocupante es que sólo se reporta el estado actual de la atmósfera en un momento dado y los planes de contingencia (tabla 4) se implementan cuando ya se ha respirado el contaminante fuera de la norma, careciéndose de acciones preventivas.

Tabla 4. Fases de contingencia ambientales e Índice Metropolitano de Calidad del Aire

\begin{tabular}{cccc|cc}
\hline Fase & Contaminante & $\begin{array}{c}\text { Activación } \\
\text { (IMECA) }\end{array}$ & $\begin{array}{c}\text { Desactivación } \\
\text { (IMECA) }\end{array}$ & \multicolumn{2}{|c}{ Escala IMECA } \\
I & $\mathrm{O}_{3} \mathrm{y} \mathrm{PM}_{10}$ & $150-200$ & Menor a 140 & 0 a 100 & Bueno \\
II & $\mathrm{O}_{3} \mathrm{y} \mathrm{PM}_{10}$ & $200-250$ & Menor a 200 & $51-100$ & Regular \\
& & & $101-150$ & Mala \\
III & $\mathrm{O}_{3} \mathrm{y} \mathrm{PM}_{10}$ & Mayor a 250 & Menor a 250 & $151-200$ & Muy Mala \\
& & & $>200$ & Extremadamente mala \\
\hline
\end{tabular}

IMECA: Índice Metropolitano de Calidad del Aire Fuente: www.semades.jalisco.gob.mx

Así, las medidas de prevención son limitadas ya que no se tiene un sistema basado en el pronóstico de las inversiones térmicas, por lo que la salud de la población presenta alta vulnerabilidad a la concentración de contaminantes en el aire. 


\section{BIBLIOGRAFÍA}

Alfaro, B., Limón R., Martínez T., Ramos, G., Reyes, A. y Tijerina, M. (2008): Ciencias del ambiente. Ed. Patria. 363 pp. México.

Borja-Aburto, V.H., Loomis, D.P., Bangdiwala, S.I., Shy, C.M., Rascón-Pacheco, R.A. (1997): «Ozone, suspended particles, and daily mortality in Mexico City», en Am J Epidemiol 145: 258-268.

CÉSPEDES, R. (2005): Impacto de la contaminación atmosférica en la salud (ICAS). Proyecto Aire Limpio: Evaluación del efecto de la contaminación atmosférica en la salud de la población del Municipio Cercado Cochabamba - Bolivia. 25 pp.

Dockery, D.W., Speizer, F.E., Stram, D.O., et al. (1989): «Effects of inhalable particles on respiratory health of children», en Am Rev Respir Dis 1989; 139: 587-594.

Enger, Eldon D. et al. (2006): Ciencia Ambiental. Un estudio de interrelaciones. Décima edición. McGraw-Hill Companies, Inc. New York, USA.

Generalitat Valenciana (2010): Red de vigilancia de la contaminación atmosférica. Calidad del aire de la Comunidad Valenciana. Conselleria de Infraestructuras, Territori i Medi Ambient (CITMA): Disponible en el enlace http://www. cma.gva.es/cidam/emedio/atmosfera/.

Martín Vide, J. (1991): Fundamentos de climatología analítica. Síntesis. 1ª edición. Madrid, 100 pp.

Meulenert A.R., Cornejo V.M., Ramírez H.U., García M.E., García F.O., Alcalá J. (2006): Análisis de las inversiones térmicas en la ZMG y su relación con los índices de contaminación. Memorias XI Congreso Internacional y Nacional de Ciencias Ambientales. Oaxtepec, Morelos. 07-09/06/2006 (en CD).

Molina L. T., Molina M. J. (2005): La calidad del aire en la megaciudad de México. Un enfoque integral. Fondo de Cultura Económica, 2005. 463 páginas. México.

Nebel B. J., Wright R. T. (1999): Ciencias ambientales. Ecología y desarrollo sostenible. Pearson - Prentice Hall. 720 páginas. México.

Nicod, Ch. y Lizuka, M. (2000): Conciencia ciudadana y contaminación atmosférica en América Latina. Ed. Comisión Económica para América Latina y el Caribe (CEPAL) y Gobierno de Japón. pp. 35 (7-8).

ProAire (2011-2020): Programa para Mejorar la Calidad del Aire Jalisco 2011 2020. Inventario de emisiones, año base 2005. 231 pp. Disponible en: www. jalisco.gob.mx.

Ramírez, H., Andrade, M., Bejaran, R., García, M., Wallo, A., Pompa, A. y De LA Torre, O. (2009): «The spatial-temporal distribution of the atmospheric polluting agents during the period 2000-2005 in the Urban Area of Guadalajara, Jalisco, Mexico», en Journal of Hazardous Materials. Vol. 165, Issues 1-3, 15 June 2009, pp. 1128-1141. 
Ramírez, H., Andrade, M., De la Torre, O., García, M., Meulenert, A. García O. y Alcalá, J. (2008): Evaluación de eventos climáticos extremos y su impacto en la salud en América Latina. Universidad de Guadalajara, México. 180 pp.

Ramírez, H., Andrade, M., GonzÁlez, M., Celis, A. (2006): «Contaminantes atmosféricos y su correlación con infecciones agudas de las vías respiratorias en niños de Guadalajara, Jalisco», en Salud Pública de México. vol. 48, núm. 005, pp. 385-394.

Schwartz J., Marcus A. (1990): Mortality and air pollution in London. A time series analysis, en Am J Epidemiol; 131:185-194.

Schwartz, J., Slater, D., Larson, T.V., et al. (1993): «Particulate air pollution and hospital emergency room visits for asthma in Seattle», en Am Rev Respir Dis 1993; 147: 826-831.

SEGala, C. (1999): Health effects of urban outdoor air pollution in children. Current epidemiological data. Pediatr Pulmonol. Vol. 18:6-8.

Semades (2012): Sistema de Monitoreo Atmosférico de Jalisco. Secretaría de Medio Ambiente para el Desarrollo Sustentable. Estado de Jalisco, MX. Disponible en los enlaces: www.semades.jalisco.gob.mx y http://siga.jalisco.gob.mx/aire/ Semarnap/Ss/Gej (1997): Programa para el mejoramiento de la calidad del aire en la ZMG, 1997-2001. Secretaría del medio ambiente, recursos naturales y pesca - Secretaría de Salud - Gobierno del Estado de Jalisco, 240 p.

Zmvм (2005): Informe Climatológico Ambiental del Valle de México. Capítulo 3: Inversiones térmicas en la ZMVM. Ed. Zona Metropolitana del Valle de México Secretaría del Medio Ambiente. Gobierno del Distrito Federal, pp. 60-84. 